\title{
Demanda residencial por energia elétrica no Brasil: uma análise econométrica a partir de séries temporais (1990-2013)
}

\section{Calisto Rocha Oliveira Neto ${ }^{1}$ Elaine Carvalho de Lima²}

\begin{abstract}
Resumo:
Com base na Teoria Econômica do Consumidor, o presente artigo busca analisar o comportamento da demanda por energia elétrica da classe residencial, entre 1990 e 2013, relacionando as variáveis Produto Interno Bruto (PIB), índice de preço dos eletrodomésticos e a tarifa da energia. Foram utilizados modelos econométricos dinâmicos com base em séries temporais como VAR e VEC, além do teste de causalidade de Granger, os quais permitem fazer previsões. As estimações sugerem que os dados se ajustam bem à teoria, uma vez que os sinais das elasticidades preço e renda do consumo residencial estão de acordo com a teoria econômica. Constatou-se que a demanda por energia vem aumentando ao longo do tempo, principalmente com crescimento PIB brasileiro.
\end{abstract}

Palavras-chave: Energia; Teoria do Consumidor, Modelos VAR e VEC;

Residential demand for electricity in Brazil: an analysis econometric from time series (1990-2013)

\begin{abstract}
:
Based on the Economic Theory of Consumer, the behavior of the demand for electricity of residential class between 1990 and 2013 is related variables Gross Domestic Product (GDP) price index of household appliances and energy rate. We used dynamic econometric models from time series, such as VAR and VEC beyond the Granger causality test, which allows making predictions. Estimates suggest that the data fit well the theory, since the signals of price and income elasticities of residential consumption are consistent with economic theory. It was found that the demand for energy is increasing over time, especially with Brazilian GDP growth.
\end{abstract}

Keywords: Energy, Consumer Theory, Modelos VAR and VEC;

Classificação JEL: C01, D11

1 Mestre em Economia pela Universidade Federal do Rio Grande do Norte. E-mail: calisto_neto@ hotmail.com

2 Doutoranda em Economia pela Universidade Federal de Uberlândia. E-mail: elaine.alirn@gmail. com 


\section{Introdução}

Historicamente, o homem, desde os seus primórdios, quase sempre dependeu de algum tipo de fonte energética que garantisse a sua sobrevivência, o desenvolvimento e a evolução de sua espécie. Assim, a obtenção e manipulação de algum tipo de fonte de energia pelo homem transformaram o seu comportamento e suas formas de interação no meio ambiente e social.

Nesse sentido, a energia elétrica cumpre um papel fundamental para o crescimento econômico e desenvolvimento social. A sociedade moderna depende cada vez mais do fornecimento de energia para assegurar a capacidade produtiva e para manter o padrão de produção capitalista com objetivo de levar conforto, bem-estar doméstico e garantir a expansão da produção de bens e serviços.

Assim, na configuração da estrutura produtiva capitalista, seus ciclos de desenvolvimento estão estritamente ligados a escolhas, direcionados e levados a cabo na conformação da estrutura energética. Isso implica que a estrutura produtiva existe e se reproduz devido à estrutura energética. Esta, por conseguinte, existe e se reproduz graças à estrutura produtiva (SILVA, 2006). Dessa forma, conclui-se que a produção e consumo processam-se pela relação mútua entre estrutura produtiva e estrutura energética.

Sendo assim, as recentes preocupações a nível mundial com a segurança energética devido ao eminente fim do petróleo e com a preservação do meio ambiente vêm determinando uma nova condição de planejamento para a expansão do setor elétrico tanto a nível mundial quanto nacional.

Em termos do sistema brasileiro de geração de energia elétrica, este tem tradição em produzir eletricidade por fonte hidrológica. A estrutura de produção de energia com base em hidrelétricas chega a superar os $61 \%$ da oferta total, seguido por termoelétricas com $26,7 \%$, energia eólica com $7,1 \%$ e $7 \%$ de outras fontes (ANEEL, 2017). É uma composição diferenciada e privilegiada quando comparada à matriz elétrica de outros países. Esse perfil dá ao Brasil uma matriz elétrica ímpar, que garante a oferta de eletricidade com características de ser menos poluente, renovável e competitiva economicamente, uma vez que o insumo água é um dado da natureza.

Contudo, o sistema hídrico apresenta forte sazonalidade com o regime de chuvas, o que gera incertezas no abastecimento. Atualmente, o atendimento à demanda só é possível devido à estratégia de construção de grandes reservatórios d'água, que tem a capacidade de controlar a oferta de eletricidade durante o ano inteiro. Todavia, questões geográficas e ambientais estão restringindo a expansão de construção de novas hidrelétricas com grande capacidade de armazenar água devido à legislação ambiental mais rígida e, também, à conscientização da importância de se preservar os recursos naturais.

Dessa forma, o objetivo deste artigo é realizar uma análise empírica sobre os determinantes do comportamento da demanda nacional por energia elétrica da 
classe residencial por meio da relação causal entre: a variável demanda por energia e as variáveis Produto Interno Bruto (PIB), tarifa de energia e o preço médio dos eletrodomésticos. Desse modo, a hipótese econômica de que o consumo de energia elétrica residencial tem correlação positiva com a expansão do Produto Interno Bruto (PIB) e negativa com a tarifa de energia e com os preços dos eletrodomésticos é corroborada pelo objetivo de expandir a economia brasileira para os próximos anos, tendo em vista o fortalecimento da dinâmica do mercado interno e expansão do número de empregos, o que provavelmente levará ao aumento da demanda por energia elétrica.

Além disso, o Novo Modelo do Setor Elétrico brasileiro, introduzido em 2004, tem como objetivo garantir a oferta de energia, promover a modicidade tarifária e permitir que brasileiros de baixa renda tenham acesso à energia elétrica especialmente pelos programas de universalização da eletricidade, como o programa luz para todos, bem como os programas sociais de distribuição de renda (ANEEL, 2008). Assim, a população mais pobre do país passou a ter acesso, não somente à energia elétrica, mas também à compra de eletrodomésticos, consequências dos programas de transferência de renda condicionada implantados nos últimos anos pelo governo federal.

O presente trabalho está estruturado em quatro seções, além da introdução. $\mathrm{Na}$ seção seguinte, é apresentada uma fundamentação teórica sobre o setor elétrico nacional. Na terceira seção, destaca-se a metodologia utilizada para obtenção e análise dos dados, em seguida, temos os principais resultados e discussões. E, por fim, as considerações finais.

\section{Referencial teórico: breve análise do setor elétrico brasileiro}

A base da matriz elétrica nacional está centrada em hidrelétricas. Atualmente, a produção de eletricidade por fonte hidrelétrica chega a aproximadamente $61 \%$ do total da produção em eletricidade. O país ocupa a segunda posição no mundo em produção de eletricidade por fonte hídrica, ficando atrás da China e a frente dos EUA. Porém, quando se compara a matriz elétrica interna desses países, o Brasil tem uma composição ímpar e privilegiada. Segundo o relatório da U.S. Energy Information Administration (U.S. EIA), em 2013, a China apresentou uma matriz elétrica baseada em fontes primárias, composta por $69 \%$ combustíveis fósseis; $22 \%$ hidroeletricidade e $9 \%$ são de outras fontes (U.S. EIA, 2015). Os EUA também têm uma matriz elétrica estruturada em combustíveis fósseis. A matriz elétrica dos EUA, em 2014, era composta por $72 \%$ de fontes primárias, $9 \%$ de hidrelétricas, $9 \%$ nuclear, $8 \%$ fontes renováveis e $2 \%$ outras fontes (U.S. EIA, 2014).

Assim, o perfil da matriz elétrica brasileira é extremamente estratégico, pois, além de garantir a oferta de eletricidade, apresenta características econômicas e ambientais interessantes, ou seja, emite relativamente pouca quantidade de gases do 
efeito estufa, além de ser renovável e competitiva economicamente. São grandes reservatórios construídos para controlar a oferta de eletricidade durante o ano todo (CASTRO, 2003).

Nesse contexto, muitos países vêm formulando suas estratégias e execução de políticas de fomento a fontes alternativas e renováveis de energia, o Brasil, também vem apresentando políticas de desenvolvimento das fontes renováveis, principalmente energia eólica, uma vez que a construção de novas hidrelétricas com essas características tem esbarrado, crescentemente, em questionamento quanto aos impactos sociais (remanejamento de comunidades tradicionais, indígenas e das populações de entorno; perda dos laços culturais e comunitários; inundação do patrimônio tangível e locais sagrados, surgimento de doenças, insegurança e incerteza, dentre outros) e ambientais (alagamento de áreas florestais, desaparecimento do habitat da fauna e flora existente, extinção de espécies, perda da biodiversidade, tremores de terra, dentre outros) (OLIVEIRA NETO, 2016).

Historicamente, observa-se que em meados da década de 1970, o setor elétrico brasileiro teve um declínio considerável decorrente do endividamento externo, recessão econômica e, consequentemente, estagnação da demanda. As empresas do setor elétrico eram utilizadas pelo governo federal como ferramenta para combater a crise externa com objetivo de alcançar metas econômicas e políticas. Segundo Abreu (1999), entre os outros motivos para queda do setor, o principal foi o esgotamento do modelo de monopólio estatal.

No entanto, a partir de 1979, o status do endividamento externo, realizado tanto nos anos anteriores quanto nos anos posteriores a 1979, agravou-se devido, principalmente, ao aumento das taxas de juros internacionais e pelo controle das tarifas como instrumento de conter a inflação durante o período de 1982 a 1993 (ABREU, 1999). A escolha do governo brasileiro de usar as empresas estatais do setor como mecanismo de implantação de políticas de desenvolvimento no país acarretou em estagnação do setor por causa do subinvestimento.

Além disso, as instituições bancárias internacionais reduziram a destinação de recursos financeiros ao Brasil devido ao elevado risco que representava, e quando tinha uma nova operação de empréstimo os banqueiros exigiam maior spread bancário. Dessa forma, estava montado o cenário perfeito para que o Brasil passasse pelo processo de adoção das políticas neoliberais no início dos anos 1990, organizadas no tripé: desregulamentação, privatização e abertura comercial (ABREU, 1999).

Nos anos 1990, depois da implantação das ideias neoliberais, o setor elétrico nacional passou por um processo de reestruturação. O processo de privatização, com a saída do Estado como indutor do planejamento energético, foi marcado por uma introdução de enormes grupos econômicos no mercado interno, resultando numa alteração significativa da estrutura de mercado.

Porém, a entrada de capital estrangeiro não resultou em grandes investimentos para aumento da capacidade produtiva do setor elétrico. As reformas dos anos 1990 desarticularam o aparelho estatal e diminuíram a organização do setor, resul- 
tando na queda da capacidade de expansão do Sistema Elétrico Brasileiro (SEB) e, por consequência, da crise do racionamento de eletricidade de 2001.

O período de 2010 foi caracterizado pela alta da demanda por energia elétrica, justificada pelo impulso da retomada do crescimento econômico, que até o primeiro semestre de 2009 foi muito abalada pelos efeitos da crise financeira internacional de 2008. Aspectos macroeconômicos entre 2003 e 2014, como o aumento da renda, manutenção do crédito e expansão do mercado interno estão entre os fatores determinantes para a retomada do crescimento da economia brasileira. Além do efeito multiplicador da economia que impulsionou outras atividades econômicas.

Em termos técnicos, o domínio da geração hídrica no sistema elétrico nacional tem um aspecto relevante, a dependência no regime de chuvas, pois não há regularidade e o sistema é caracterizado por uma forte sazonalidade. Esse comportamento sazonal é ilustrado no gráfico 1 , a seguir.

Gráfico 1: Energia Natural Afluente: média história anual. Inclui a produção de todos os subsistemas do SIN* (configuração de 2010)

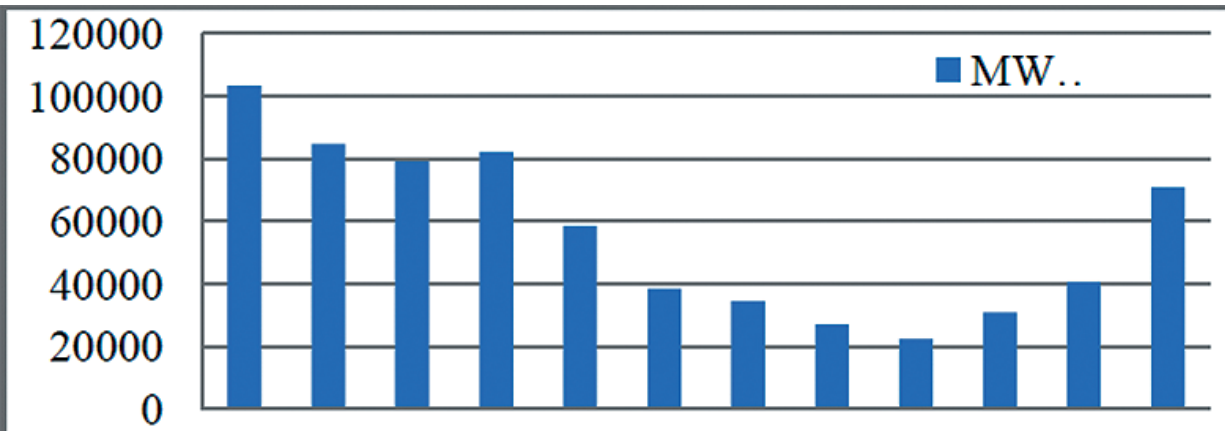

Jan Fev Mar Abr Mai Jun Jul Ago Set Out Nov Dez

(*) Sistema Interligado Nacional que corresponde a algo em torno de $98 \%$ do mercado brasileiro de energia elétrica

Fonte: Elaboração própria com base nos dados do ONS (www.ons.org.br)

De acordo com o gráfico 1, é possível observar que no mês de janeiro a Energia Natural Afluente (ENA) ${ }^{3}$ ultrapassa os 100 mil MWmed ao contrário do que ocorre com a ENA no mês de setembro que chegou a produzir apenas 23 mil MWmed $^{4}$.

Energia hídrica que corre pelos rios com fins energéticos.

4 Estes números incluem apenas os rios que já possuem aproveitamento hidroelétrico. 


\subsection{Demanda Residencial de Energia Elétrica: uma abordagem empí- rica}

Diversos trabalhos calcularam, ao longo do tempo, a demanda de energia elétrica (residencial, comercial e/ou industrial) tanto a nível estadual quanto nacional usando diferentes modelagens econométricas. Um estudo pioneiro no Brasil que abordou a questão da demanda de energia elétrica foi o de Modiano (1984), que tinha o objetivo de estudar as elasticidades-renda e preço do consumo de energia elétrica nas três classes de consumo (residencial, comercial e industrial) com base em dados anuais de 1963 até 1981.

Modiano (1984) utilizou a modelagem econométrica dos Mínimos Quadrados Ordinários (MQO), obtendo os seguintes resultados: para as classes residencial, comercial e industrial foram encontradas elasticidades preço de curto prazo de $-0,118,-0,062$, e -0,451, respectivamente. Já para as elasticidades preço de longo prazo foram encontrados valores de $-0,403,-0,183$, e -0,222. Já as elasticidades renda de curto prazo os valores encontrados foram 0,332, 0,362 e 0,502 e para o longo prazo os valores foram de 1,13, 1,068 e 1,360. Esses resultados permitem observar que o consumo seria mais elástico às variações de renda do que das tarifas aplicadas no período de análise.

Andrade e Lobão (1997), também estimaram as elasticidades renda e elasticidade preço para a classe residencial da demanda por energia elétrica para o Brasil, chegando aos valores de 0,2132 e -0,05084, respectivamente. Além disso, os autores usaram o modelo econométrico de Correção de Erros Vetoriais (VEC), modelo mais sofisticado, que permitiu aos autores fazerem previsão do consumo de energia elétrica residencial para o período de 1997 a 2005.

Outro estudo interessante sobre a demanda nacional de energia foi feito por Saed (2004), que estimou a demanda da Jordânia entre 1980 e 1990. O autor chegou à conclusão de que o consumo de energia está correlacionado positivamente com o crescimento da economia e correlacionado negativamente com o preço da tarifa. Ou seja, o consumo de energia aumenta quando há expansão do PIB (elasticidade renda positiva) e cai quando o preço da tarifa aumenta (elasticidade preço negativa).

Por seu turno, Irffi et al (2009) realizaram uma análise para a região Nordeste com dados anuais para o período de 1970 a 2003, os autores utilizaram como métodos os Mínimos Quadrados Dinâmico (MDQ) e Mudança de Regime (MR), estimou-se o modelo para as classes industrial, comercial e residencial. Os resultados obtidos foram comparados com as elasticidades-preço e renda de curto e longo prazo com outros estudos a nível de Brasil, tais como, da Eletrobrás e Siqueira, Cordeiro Jr. e Castelar (2006). De modo geral, as previsões resultantes foram mais apuradas que estudos anteriores.

Por fim, Mattos e Lima (2005) estimaram a demanda residencial de eletricidade do estado de Minas Gerais (MG) entre os anos 1970 e 2002. Foram estimadas as elasticidades preço e renda da demanda por energia residencial de MG. Como 
método, utilizou-se o Modelo de Correção de Erros Vetoriais (VEC) para estimação, optando por utilizar o conceito de cointegração depois de identificar que as séries analisadas eram não estacionárias. De acordo com os autores, os resultados mostraram que a demanda por energia era mais sensível a variações da renda quando comparado ao preço da energia elétrica, muito embora tanto a elasticidade renda quanto a elasticidade preço tiveram resultados inferiores a uma unidade. Isto é, a cada aumento de uma unidade monetária na renda a demanda por energia cresce mais do que quando há queda no preço da tarifa de energia.

Nestes termos, na Teoria Microeconômica do Consumidor tendo como referência a teoria da Utilidade, o consumidor busca a maior satisfação de um bem ou serviço perante a um conjunto de cestas de consumo disponíveis (VARIAN, 2006). Sendo assim, para o caso deste estudo, a demanda por energia elétrica residencial pode ser caracterizada, a priori, como um problema de maximização da utilidade, em que o consumidor está sujeito a uma restrição orçamentária. Esta abordagem difere da demanda comercial e industrial por energia elétrica, pois a Teoria Microeconômica descreve que o produtor tem o objetivo de maximizar receita e minimizar custo, sujeito a certo nível de venda e/ou produção no mercado.

Conceitualmente, pode-se dizer que, a elasticidade renda da demanda por energia elétrica do consumidor é o resultado do quanto de expansão na produção de energia elétrica é necessária para suportar um aumento na renda ou PIB. Enquanto que, a elasticidade preço reage positivamente a uma queda da tarifa e dos preços dos eletrodomésticos. Ou seja, na Teoria Microeconômica a elasticidade renda e elasticidade preço da demanda por energia elétrica medem a variação, em percentagem, da quantidade demandada de energia resultante do desempenho positivo do PIB e da queda no preço da tarifa e dos preços dos eletrodomésticos.

De acordo com Varian (2006), a demanda de um dado bem $i$ pode ser representada da seguinte maneira:

Maximizar $U\left(X_{i}\right)$

Sujeito a: $P_{i} X_{i}+P_{n} X_{n} \leq R$

Onde: $U\left(X_{i}\right)$ é a função de utilidade do consumidor; $P_{i}$ é o preço do bem $i$; é o preço de todos os $n$ bens consumidos; $X_{i}$ é a quantidade consumida do bem $i ; X_{n}$ é a quantidade consumida dos $n$ bens; $R$ é a renda do consumidor.

\section{Metodologia e modelo econométrico}

A metodologia de série de tempo permite mostrar historicamente a sucessão de dados aleatórios observados em períodos de tempo homogêneos, ou seja, a observação dos dados pode ser coletada diariamente, semanalmente, mensalmente, trimestralmente, anualmente, etc. A suposição que sustenta a análise de uma série temporal é a possibilidade de se fazer previsão. 
Neste trabalho, que analisa a demanda por energia elétrica na classe residencial em função do PIB, do preço da tarifa e do índice de preço dos eletrodomésticos, foram utilizados os modelos de Vetor Autorregressivo (VAR), o teste de causalidade de Granger e Vetor de Correção de Erros (VEC) de série temporal para analisar o comportamento do consumo de energia elétrica para o período de janeiro de 1990 até junho de 2013.

Descrito a forma funcional das variáveis, seguem os procedimentos iniciais da metodologia de análise da série temporal para este trabalho. O primeiro procedimento é fazer inferência sobre a estacionariedade da série. Depois, constata-se se há presença ou não de quebra estrutural na análise da série, procedimento justificado, neste trabalho, pela crise energética nos anos 2001 e 2002 no Brasil. Para alcançar esses dois objetivos iniciais foram feitos o teste de raiz unitária de Duckey-Fuller Aumentado (ADF) e, em seguida, o teste Philipps-Perron para verificar se há uma mudança estrutural no padrão de consumo de energia elétrica, esses procedimentos estatísticos são muito utilizados em séries temporais.

$\mathrm{Na}$ sequência, verifica-se, por meio dos modelos VAR-VEC, o número ótimo de defasagens para estimar o modelo. No passo seguinte, faz-se o teste de cointegração das variáveis pelo método de cointegração de Johansen (1988), que permite verificar se as variáveis possuem uma relação constante e estável ao longo do tempo.

O modelo de Vetor Autorregressivo (VAR) é uma modelagem que usa equações multivariadas que permite analisar a existência de relações de interdependência entre os sistemas de equações em análise, considerando-as como endógenas (em defasagem) para mensurar o impacto que estas têm nas variáveis no presente, ou seja, na análise de regressão o método VAR permite modelar a variável em termos dos seus valores passados da série. Além disso, sinaliza possíveis impactos de perturbações estocásticas na série de tempo. Essas características permitem ao modelo observar o comportamento da série no futuro, ou seja, o VAR é bastante eficiente em análise de previsão (GUJARATI, 2006).

Já o modelo VEC possibilita ver as relações de curto prazo entre a demanda por energia e os regressores da estimação. A estimação desse modelo faz-se depois de estimar o modelo VAR incorporando a correção do erro do modelo VAR. A vantagem da utilização do Vetor de Correção de Erros é que ele permite verificar as dinâmicas de curto e longo prazos das séries com as estimativas das elasticidades. A decisão de utilizar um Vetor de Correção de Erros (VEC), neste estudo, foi por causa da não estacionariedade de primeiro nível. Assim, a ferramenta VEC fornece o mais sofisticado tratamento estatístico e econométrico, tanto para estimar a função demanda de longo prazo quanto para realizar previsões de consumo futuro de energia elétrica.

Contudo, quando a série for não estacionária a nível zero I(0), ou seja, se não apresentar média e variância constantes ao longo do tempo, bem como for cointegrada de mesma ordem, a estimação pelo método VAR não é mais apropriada à análise inicial. Sendo assim, pelo teorema de Engle e Granger (1987), a existência 
de raízes unitárias nas séries não impede, a priori, fazer modificações, já que, com o método de cointegração a relação torna-se estatisticamente significante.

Como especificado anteriormente, um ponto importante na estimação dos modelos VAR-VEC diz respeito à conceituação do número de defasagens em que o modelo será estimado. No presente trabalho, será utilizado o número de defasagens de acordo com o maior número de critérios: Erro Final de Previsão (FPE), Akaike (AIC), Teste Estatístico Sequencial Modificado (LR), Hannan-Quinn (HQ) e Schwarz (SIC).

Para a estimação do modelo mencionado, foi considerado o período de janeiro de 1990 a junho de 2013, a seleção desse período de análise justifica-se pelas mudanças estruturais na economia do Brasil, tais como: a abertura econômica e as políticas de cunho liberal, a implementação do Plano Real, a crise energética de 2001, a universalização do acesso à energia e a expansão dos programas de transferência de renda, que possibilitaram um maior poder aquisitivo, logo, maior acesso ao consumo.

Como se trata da demanda de energia da classe residencial, o presente trabalho busca verificar a evolução do consumo dessa classe relacionando com o comportamento da economia e as políticas sociais do Estado brasileiro, principalmente pós-racionamento de energia de 2001. Esse racionamento de energia elétrica entre 2001 e 2002 teve impacto direto no consumo, pois foi preciso reduzi-lo por causa do Programa de Racionamento de energia elétrica. Bardelin (2004) destaca outros fatores que contribuíram para a redução do consumo, como: reajustes nas tarifas de energia elétrica; redução de impostos sobre equipamentos com maior eficiência energética; elevação dos impostos que incidem sobre os equipamentos de menor eficiência.

Contudo, quando se analisa o comportamento do consumo pós-racionamento, observa-se um crescimento no consumo de energia elétrica da classe residencial. Em valores absolutos, o consumo de energia pelas residências passou de $6.786 \mathrm{GWh}$ em 2003 para 8.893 GWh em 2011 (ELETROBRÁS, 2011).

Segundo a Empresa de Pesquisa Energética (EPE), a expansão do consumo residencial no Brasil, principalmente, a partir de 2003, explica-se pelo aquecimento da economia brasileira que reflete em mais emprego e renda e pela oferta de crédito, que estimula a compra e uso de aparelhos eletroeletrônicos. Ademais, destacam-se os impactos positivos dos programas sociais do governo na expansão da demanda agregada via consumo, como os programas Bolsa Família e o Luz para Todos. Esses programas permitiram a uma parcela da população do país ter acesso ao mercado consumidor.

O modelo matemático que pode representar uma função de demanda por energia elétrica ao longo do tempo é uma função Coubb-Douglas, temos:

$$
\mathrm{C}_{\mathrm{t}}=\alpha_{0} \mathrm{P}_{\mathrm{t}}^{\varphi} \mathrm{Y}_{\mathrm{t}}^{\beta} \mathrm{E}_{\mathrm{t}}^{\delta}
$$


$\alpha_{0}>0, \varphi<0, Y>0$, e $<0$, que tomando o seu logaritmo em ambos os lados, chega-se à seguinte equação linear de demanda por energia elétrica:

$$
\log C t=\log \alpha+\log \phi \mathrm{Pt}+\log \beta \mathrm{Yt}+\log \delta \mathrm{Et}
$$

Em que,

$\mathrm{C}_{\mathrm{t}}$ é o consumo residencial de energia elétrica no tempo $t$;

$\mathrm{P}_{\mathrm{t}}$ é a tarifa residencial de energia elétrica no tempo $t$;

$\mathrm{Y}_{\mathrm{t}}$ é o desempenho da renda (PIB) no tempo $t$;

$\mathrm{E}_{\mathrm{t}}$ é o índice de preço dos aparelhos eletrodomésticos no tempo $t$;

Em que a quantidade demandada por energia seria função das três variáveis explicativas (renda, preço da tarifa e preço dos eletrodomésticos):

$$
\mathrm{C}_{\mathrm{t}}=f\left(\mathrm{Y}_{\mathrm{t}} ; \mathrm{P}_{\mathrm{t}} ; \mathrm{E}_{\mathrm{t}}\right)
$$

Quadro 1- Descrição dos dados da pesquisa

\begin{tabular}{|ccc|}
\hline \multicolumn{2}{|c|}{ Variáveis (séries mensais de jan/1990 a jun/2013) } & Fonte \\
\hline CR & Consumo residencial total (em MWh) & Eletrobrás in IPEA \\
TAR & Tarifa residencial (em R\$ por MWh) & Eletrobrás in IPEA \\
PIB & Produto interno bruto (em milhões de R\$) & (BCB Boletim/Ativ. Ec.) in IPEA. \\
& Índice de preço dos eletrodomésticos - & Deflator IPA-EP geral (FGV/Conj. \\
E & Índice (ago. 1994=100) & Econ. - IGP) in IPEA \\
\hline
\end{tabular}

Fonte: Elaboração própria

\section{Resultados e discussões}

O comportamento da demanda residencial por energia entre 1990 e 2013 pode ser explicitado pelo gráfico 2 abaixo. Nota-se que o consumo de energia elétrica tem uma tendência de crescimento ao longo do tempo sem apresentar volatilidade na demanda, a exceção foi a crise estrutural e climática apresentada pelo setor elétrico no ano de 2001, no gráfico vê-se a queda no consumo. Alguns autores afirmam que a principal causa da crise, além da falta de chuva naquele ano, foi a falta de investimentos decorrentes da saída do Estado como planejador do sistema elétrico a partir do início dos anos 1990 com a introdução das políticas neoliberais, em que o capital privado passaria a investir no setor. 
Gráfico 2: Consumo residencial de eletricidade (1990-2013)

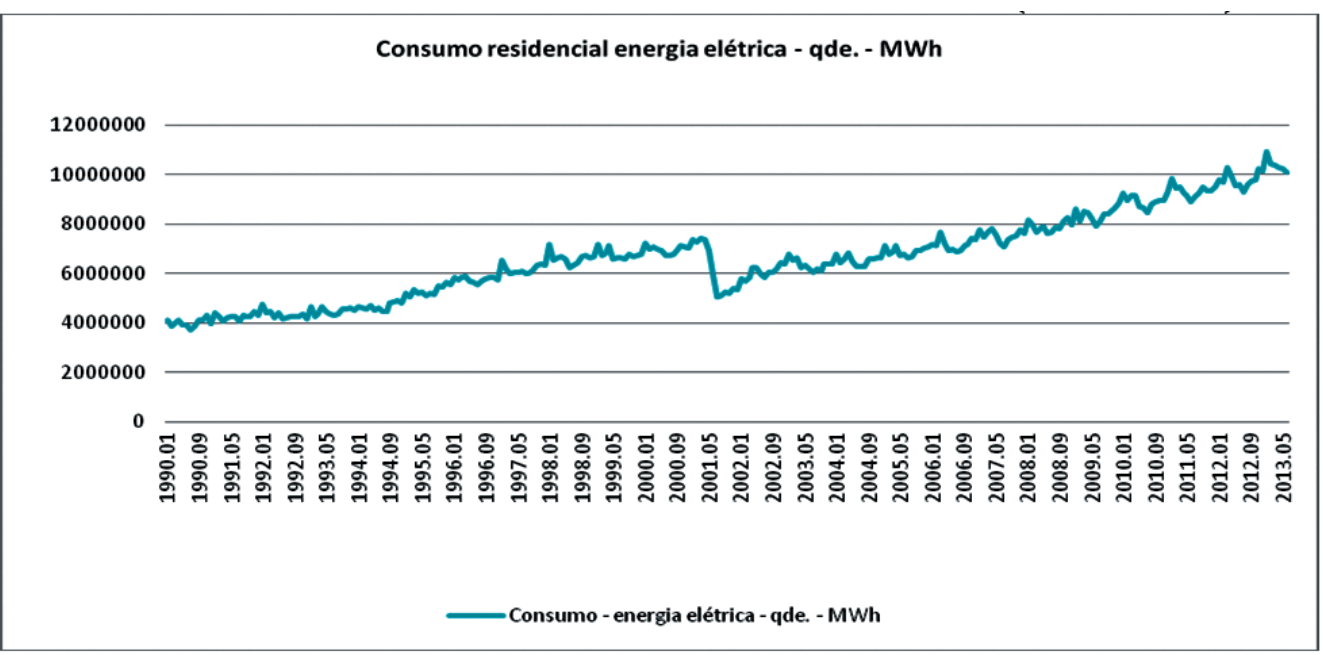

Fonte: Elaboração própria

Em seguida, serão analisados os resultados estimados para a demanda de energia elétrica residencial por meio dos modelos VAR-VEC e depois as elasticidades preço e renda da demanda por eletricidade foram estimadas. Como se trata de análise de série temporal é preciso identificar a ordem de integração da série.

Sendo assim, é necessário primeiramente calcular a estacionariedade da série consumo por meio do Teste de Raiz Unitária de Dickey-Fuller Aumentado (DFA). $\mathrm{Na}$ sequência, compara-se a estatística do teste DFA com valores críticos a 1\%, 5\% e $10 \%$ da tabela estatística Tau $(\tau)$ para saber se pode ou não fazer previsão do consumo de energia. Os resultados estão na tabela 2, a seguir, e observa-se que, para as quatro variáveis (consumo, PIB, tarifa e índice de preços dos eletrodomésticos) há presença de raiz unitária aos três níveis de significância (1\%, $5 \%$ e $10 \%)$, pois os valores calculados (DFA), em módulo, são menores do que o valor crítico (tabelado) do teste, logo, a série caracteriza-se como não estacionária em nível.

Dessa forma, a série possui um passeio aleatório e precisa-se fazer a diferenciação com o objetivo de torná-la estacionária para todas variáveis, logo, fez-se o teste em primeira diferença ou I(1). Verificou-se que a série torna-se estacionária. $\mathrm{Na}$ tabela 1, observa-se que, quando se faz a diferenciação, os valores calculados, em módulo, passam a ser maiores do que o valor crítico da estatística $\tau$. 
Tabela 1: Testes de Dickey-Fuller Aumentado (DFA) para as séries Consumo (C), PIB, Tarifa e Índice de Preços Eletrodomésticos

\begin{tabular}{|c|c|c|c|c|c|c|}
\hline \multirow{2}{*}{ Variáveis } & \multirow{2}{*}{$\begin{array}{l}\text { Forma do } \\
\text { teste }\end{array}$} & \multirow{2}{*}{$\begin{array}{l}\mathrm{N}^{\circ} \text { de defa- } \\
\text { sagem }\end{array}$} & \multirow{2}{*}{$\begin{array}{l}\text { Teste } \\
\text { DFA }\end{array}$} & \multicolumn{3}{|c|}{ Valores Críticos } \\
\hline & & & & $1 \%$ & $5 \%$ & $10 \%$ \\
\hline $\mathrm{C}$ & $\begin{array}{c}\text { Constante e } \\
\text { sem tendência }\end{array}$ & 1 & -1.0832 & -3.4535 & -2.8716 & -2.5722 \\
\hline$\Delta \mathrm{C}$ & $\begin{array}{c}\text { Constante e } \\
\text { tendência }\end{array}$ & 0 & -21.9929 & -3.9911 & -3.4259 & -3.1361 \\
\hline PIB & $\begin{array}{c}\text { Constante e } \\
\text { sem tendência }\end{array}$ & 2 & -2.7517 & -3.9912 & -3.4260 & -3.1361 \\
\hline$\Delta \mathrm{PIB}$ & $\begin{array}{c}\text { Constante e } \\
\text { tendência }\end{array}$ & 1 & -8.6322 & -3.9912 & -3.4260 & -3.1361 \\
\hline Tarifa & $\begin{array}{c}\text { Constante e } \\
\text { sem tendência }\end{array}$ & 0 & -1.6589 & -3.4534 & -2.8715 & -2.5722 \\
\hline$\Delta$ Tarifa & Nenhuma & 0 & -14.9307 & -2.5732 & -1.9419 & -1.6159 \\
\hline $\begin{array}{c}\text { Índice de } \\
\text { PE }\end{array}$ & Nenhuma & 1 & -0.0454 & -2.5732 & -1.9419 & -1.6159 \\
\hline $\begin{array}{l}\Delta \text { Índice } \\
\text { de PE }\end{array}$ & $\begin{array}{l}\text { Constante e } \\
\text { sem tendência }\end{array}$ & 0 & -5.5107 & -3.4535 & -2.8716 & -2.5722 \\
\hline
\end{tabular}

*No teste Dickey-Fuller Aumentado (DFA), foi utilizado o número de defasagens que minimizava o Critério de Informação de Schwarz.

Fonte: Dados da pesquisa

Destaca-se, também, a presença de quebra estrutural no consumo de energia elétrica ocorrida entre os anos de 2001 e 2002 (ver gráfico 1), devido ao racionamento de energia elétrica. Isso poderia levar o teste DFA a concluir levianamente a presença de uma raiz unitária. Dessa forma, utilizou-se o teste de raiz unitária de Philipps-Perron, que leva em consideração a quebra estrutural na série. Esse teste incorpora uma dummy para captar a mudança estrutural. Os resultados são apresentados a seguir, na tabela 2, no qual confirmam que os resultados anteriores são $\mathrm{I}(1)$. 
Tabela 2: Teste de raiz unitária com quebra estrutural Phillips-Perron

\begin{tabular}{|c|c|c|c|c|c|c|}
\hline \multirow{2}{*}{ Variável } & \multirow{2}{*}{ Forma do teste } & \multirow{2}{*}{$\begin{array}{l}\mathrm{N}^{\mathrm{o}} \text { de defa- } \\
\text { sagem }\end{array}$} & \multirow{2}{*}{$\begin{array}{c}\text { Teste } \\
\text { P-P }\end{array}$} & \multicolumn{3}{|c|}{ Valores Críticos } \\
\hline & & & & $1 \%$ & $5 \%$ & $10 \%$ \\
\hline$\Delta \mathrm{C}$ & Constante e tendência & 11 & -25.7232 & -3.9911 & -3.4259 & -3.1361 \\
\hline$\Delta \mathrm{C}$ & Constante e sem tendência & 11 & -25.7877 & -3.4535 & -2.8716 & -2.5722 \\
\hline
\end{tabular}

\section{Fonte: Dados da pesquisa}

Na estimação dos modelos VAR-VEC, como proposto neste trabalho, os critérios LR, FPE e AIC sugeriram um modelo com oito defasagens. Já para os critérios SC e HQ, a estimação sugeriu três e quatro defasagens, respectivamente. Os resultados dos testes são apresentados a seguir na tabela 3. Como demonstra a estimação, o modelo utiliza-se de oito períodos de defasagem ótima, pois foi esse o maior número de critérios.

Tabela 3: Critérios de definição para o número de defasagens na série temporal

\begin{tabular}{c|c|c|c|c|c|c}
\hline Lag & LogL & LR & FPE & AIC & SC & HQ \\
\hline 0 & -833.8813 & NA & 0.005324 & 6.115922 & 6.168669 & 6.137093 \\
\hline 1 & 1195.690 & 3985.071 & $2.20 \mathrm{e}-09$ & -8.581680 & -8.317948 & -8.475825 \\
\hline 2 & 1473.845 & 538.0367 & $3.25 \mathrm{e}-10$ & -10.49522 & -10.02050 & -10.30468 \\
\hline 3 & 1523.787 & 95.14593 & $2.54 \mathrm{e}-10$ & -10.74297 & $-10.05727 *$ & -10.46775 \\
\hline 4 & 1554.562 & 57.73099 & $2.28 \mathrm{e}-10$ & -10.85082 & $-10.05727 *$ & $-10.49091 *$ \\
\hline 5 & 1580.080 & 47.12465 & $2.13 \mathrm{e}-10$ & -10.92030 & -9.812621 & -10.47570 \\
\hline 6 & 1599.784 & 35.81169 & $2.07 \mathrm{e}-10$ & -10.94733 & -9.628669 & -10.41805 \\
\hline 7 & 1634.798 & 62.61665 & $1.81 \mathrm{e}-10$ & -11.08612 & -9.556473 & -10.47216 \\
\hline 8 & 1660.729 & $45.61507 *$ & $1.68 \mathrm{e}-10 *$ & -11.08612 & -9.417974 & -10.45996 \\
\hline
\end{tabular}

* Número de defasagens indicado pela estimação

Onde:

LR: Sequência modificada de LR, teste estatístico ao nível de 5\%;

FPE: Erro de predição final;

AIC: Critério de Akaike;

SC: Critério de Schwarz;

HQ: Critério de Hannan-Quinn.

Fonte: Dados da pesquisa

A seguir, na tabela 4, é realizado o procedimento de cointegração de Johansen para identificação ou não da presença de um vetor de cointegração por meio dos testes do traço da matriz e dos autovalores da matriz. Assim, pelos valores calculados das estatísticas do traço e dos autovalores, as varáveis são cointegradas. Ou seja, a 
existência de causalidade multivariada entre as variáveis: Consumo de energia, PIB, Tarifa da eletricidade e Índice de preço dos eletrodomésticos sugere a existência de causalidade de Granger. E, dessa forma, elimina-se o problema da regressão espúria, podendo fazer estimações de longo prazo entre as variáveis.

Tabela 4: Testes do traço da matriz e de autovalores- Procedimento de cointegração de Johansen

\begin{tabular}{cccc}
\hline $\begin{array}{c}\text { Teste do traço da matriz } \\
\text { (rank cointegração) }\end{array}$ & Estatística do traço & Valores críticos & Prob \\
r = o & 108.1580 & 47.85613 & 0.0000 \\
r no max 1 & 55.28104 & 29.79707 & 0.0000 \\
r no max 2 & 24.21005 & 15.49471 & 0.0019 \\
r no max 3 & 4.142277 & 3.841466 & 0.0418 \\
Teste dos autovalores & Estatística do traço & Valores críticos & Prob \\
r = 0 & 52.87698 & 27.58434 & 0.0000 \\
r no max 1 & 31.07099 & 21.13162 & 0.0015 \\
r no max 2 & 20.06778 & 14.26460 & 0.0054 \\
r no max 3 & 4.142277 & 3.841466 & 0.0418 \\
\hline
\end{tabular}

Fonte: Dados da pesquisa

Dessa forma, a causalidade de Granger pode ser analisada com base na estimação do vetor de cointegração com objetivo de observar os efeitos de longo prazo entre as variáveis. Ver a tabela 5, abaixo.

Tabela 5: vetor de cointegração para a variável consumo de energia

\begin{tabular}{cccc}
\hline Consumo & $\begin{array}{c}\text { Índice de preço } \\
\text { eletr. }\end{array}$ & PIB & Tarifa \\
$\mathbf{1 . 0 0 0 0 0 0}$ & 0.0000 & 0.3303 & -0.6352 \\
& & $(0.08969)$ & $(0.09513)$ \\
& & {$[-2.06313]$} & {$[3.59127]$} \\
\hline
\end{tabular}

Os valores entre parênteses são os Erros-padrão

*Os valores entre colchetes são as Estatísticas $t$

Fonte: Resultados da pesquisa 
Diante do resultado do vetor de cointegração estimado, pode-se deduzir uma relação de equilíbrio de longo prazo da variável consumo residencial de eletricidade pela equação abaixo:

$\mathrm{Cr}=0.6352 \mathrm{PIB}-0.3303 \mathrm{TAR}$

em que,

Cr: Consumo de energia residencial

PIB: Produto Interno Bruto

TAR: Tarifa da energia elétrica

Assim, a equação estimada está de acordo com a teoria econômica, pois as relações entre as variáveis apresentam sinais esperados de acordo com a demanda do consumidor, ou seja, há uma relação positiva entre renda e demanda por energia e uma relação negativa entre tarifa de eletricidade e demanda por energia. Para corroborar a hipótese teórica, o teste $t$ apresenta significância estatística a 5\%. Ou seja, a estatística t calculada é maior do que o valor tabelado, logo, é possível rejeitar a hipótese nula de que os coeficientes são iguais à zero. Logo, se aceita a hipótese alternativa de que os coeficientes da equação são significativos.

Ademais, os valores dos coeficientes estimados permitem fazer interpretação econômica, ou seja, a demanda por energia elétrica é relativamente sensível à variação do PIB e pouco sensível à variação do preço da tarifa de energia. Em outras palavras, o consumo de energia em relação à renda e à tarifa são inelásticas. Em termos econômicos, os valores dos coeficientes mostram que um aumento de $1 \%$ no PIB causa um aumento na demanda por energia elétrica na ordem de 0,63\%, e uma queda de $1 \%$ no preço da tarifa causa um aumento na demanda por eletricidade na ordem de $0,33 \%$.

Por fim, depois das estimações e análises dos testes de raiz unitária e do vetor de cointegração, pode-se estimar o modelo VEC com objetivo de determinar o comportamento da demanda por energia no futuro. Dessa forma, como a série é cointegrada, para especificar de forma correta a relação entre consumo de energia e as variáveis explicativas (PIB, tarifa e índice de preço de eletrodomésticos) torna-se necessário incorporar ao modelo o desequilíbrio de curto prazo dos valores da série consumo em relação ao equilíbrio de longo prazo. Para isso, é preciso incluir os valores defasados dos resíduos no modelo expresso em primeiras diferenças. Ver tabela 6 abaixo. 
Tabela 6: Estimação do modelo VEC para todas as variáveis

\begin{tabular}{|c|c|c|c|c|}
\hline Variáveis explicativas & $\begin{array}{c}1^{\mathrm{a}} \text { eq } \\
\mathrm{D}(\mathrm{Ct})\end{array}$ & $\begin{array}{c}2^{\mathrm{a}} \mathrm{eq} \\
\mathrm{D}(\mathrm{PIBt})\end{array}$ & $\begin{array}{c}3^{\mathrm{a}} \text { eq } \\
\mathrm{D}(\mathrm{TFt})\end{array}$ & $\begin{array}{c}4^{\mathrm{a}} \mathrm{eq} \\
\mathrm{D}(\mathrm{PEt})\end{array}$ \\
\hline \multirow{4}{*}{ Constante } & 0.003072 & 0.022803 & -0.001933 & 0.014437 \\
\hline & $(0.00282)$ & $(0.00404)$ & $(0.04215)$ & $(0.00214)$ \\
\hline & {$[1.09107]$} & {$[5.64736]$} & {$[-0.04587]$} & {$[6.74873]$} \\
\hline & {$[-1.00295]$} & {$[8.66057]$} & {$[-1.60405]$} & {$[10.5927]$} \\
\hline \multirow{3}{*}{$\mathrm{Ut}-1$} & -0.251381 & -0.201369 & -1.525698 & 0.000930 \\
\hline & $(0.06056)$ & $(0.08684)$ & $(0.90644)$ & $(0.04601)$ \\
\hline & {$[-4.15087]$} & {$[-2.31888]$} & {$[-1.68317]$} & {$[0.02022]$} \\
\hline \multirow{3}{*}{$\mathrm{Ut}-2$} & 0.059090 & 0.372800 & 0.454973 & 0.066846 \\
\hline & $(0.06244)$ & $(0.08953)$ & $(0.93452)$ & $(0.04743)$ \\
\hline & {$[0.94639]$} & {$[4.16404]$} & {$[0.48685]$} & {$[1.40933]$} \\
\hline \multirow{3}{*}{$\mathrm{Ut}-1$} & 0.043096 & 0.206916 & -0.724749 & -0.037524 \\
\hline & $(0.05113)$ & $(0.07332)$ & $(0.76536)$ & $(0.03885)$ \\
\hline & {$[0.84279]$} & {$[2.82201]$} & {$[-0.94694]$} & {$[-0.96598]$} \\
\hline \multirow{3}{*}{$\mathrm{Ut}-2$} & -0.119792 & -0.165710 & 0.355715 & -0.077992 \\
\hline & $(0.04782)$ & $(0.06857)$ & $(0.71579)$ & $(0.03633)$ \\
\hline & {$[-2.50489]$} & {$[-2.41652]$} & {$[0.49695]$} & {$[-2.14682]$} \\
\hline \multirow{3}{*}{$\mathrm{Ut}-1$} & -0.001607 & 0.001223 & 0.132975 & -0.005002 \\
\hline & $(0.00413)$ & $(0.00593)$ & $(0.06185)$ & $(0.00314)$ \\
\hline & {$[-0.38901]$} & {$[0.20644]$} & [2.14999] & {$[-1.59345]$} \\
\hline \multirow{3}{*}{$\mathrm{Ut}-2$} & -0.005603 & -0.027462 & -0.007136 & -0.019317 \\
\hline & $(0.00415)$ & $(0.00595)$ & $(0.06212)$ & $(0.00315)$ \\
\hline & {$[-1.35002]$} & {$[-4.61491]$} & {$[-0.11488]$} & {$[-6.12735]$} \\
\hline \multirow{3}{*}{$\mathrm{Ut}-1$} & -0.049630 & 0.062179 & 1.280581 & 0.690832 \\
\hline & $(0.09673)$ & $(0.13870)$ & $(1.44782)$ & $(0.07348)$ \\
\hline & {$[-0.51307]$} & {$[0.44829]$} & {$[0.88449]$} & [9.40128] \\
\hline \multirow{3}{*}{ Ut-2 } & 0.156742 & 0.412859 & 0.031404 & 0.102023 \\
\hline & $(0.08481)$ & $(0.12161)$ & $(1.26936)$ & $(0.06443)$ \\
\hline & [ 1.84820$]$ & [3.39503] & {$[0.02474]$} & [ 1.58359$]$ \\
\hline
\end{tabular}


Por fim, na tabela 7 a seguir, mostram-se os resultados do teste de causalidade de Granger. É um teste que permite observar se uma variável X "Granger-causa" uma variável Y. Em média, verifica-se a ocorrência do evento Y dado a ocorrência do evento $\mathrm{X}$ em algum período passado. $\mathrm{O}$ teste estima o melhor modelo autorregressivo, além de acrescentar quantas defasagens na equação for necessário ( $\mathrm{MO}$ RETTIN; TOLOI, 2006).

Tabela 7: Teste de causalidade de Granger

\begin{tabular}{cccc}
\hline Var. Dependente & $\mathbf{X}^{2}$ & Gl & Prob \\
\hline PIB & 6.488482 & 2 & 0.0390 \\
Tarifa & 2.089082 & 2 & 0.3519 \\
Índice PE & 1.294087 & 2 & 0.0168 \\
Resultado conjunto & 8.9154 & 6 & 0.1784 \\
\hline
\end{tabular}

Fonte: Resultados da pesquisa

Em suma, os resultados revelam que as variáveis PIB, tarifa e índice de preços dos eletrodomésticos causam no sentido Granger, a variável consumo de energia no futuro. Diferente da análise de longo prazo, os fatores que influenciam mais a determinação da demanda por energia são as variações na renda (PIB) e no preço da tarifa. Sendo a renda mais sensível na influência no consumo de energia elétrica.

A seguir, é analisada a estimação da função impulso-resposta, na qual permite traçar o efeito do choque individual das variáveis do sistema em resposta a mudanças em outras variáveis do modelo. Com isso, torna-se possível determinar a direção das respostas da demanda diante de choques na própria variável e nas demais variáveis, ou seja, tal função representa o caminho e as defasagens de tempo necessárias para que as variáveis retornem à trajetória natural. Para o presente trabalho, foi utilizada a decomposição de Cholesky que explica como os choques nas variáveis afetam as demais por meio da estrutura dinâmica do modelo (PINTO; VIEIRA, 2008).

$\mathrm{Na}$ figura 1, é possível observar a relação entre impulso e resposta das variáveis no comportamento do consumo de energia, ou seja, pode-se verificar o impacto que um choque no desvio padrão de uma variável provoca no desvio padrão de uma série de variáveis (endógenas). Por exemplo, na primeira linha de gráficos da figura 1, percebe-se que, um choque no desvio padrão do consumo (impulso) causa choques (respostas) nas demais variáveis do modelo, como PIB, tarifa de energia e preço médio do eletrodoméstico. Desse modo, como ilustrado na primeira linha dos gráficos, há uma tendência no longo prazo da demanda por energia ser explicada menos por ela mesma e mais pelo crescimento do PIB e diminuição do preço da tarifa. Ademais, constata-se que todas as variáveis são afetadas por choques não esperados no curto prazo, com exceção dos choques nos preços médios dos eletro- 
domésticos que tiveram variações quase nulas no período analisado.

Figura 1- Gráfico da função impulso-resposta das variáveis consumo, PIB, tarifa e preço médio do eletrodoméstico.
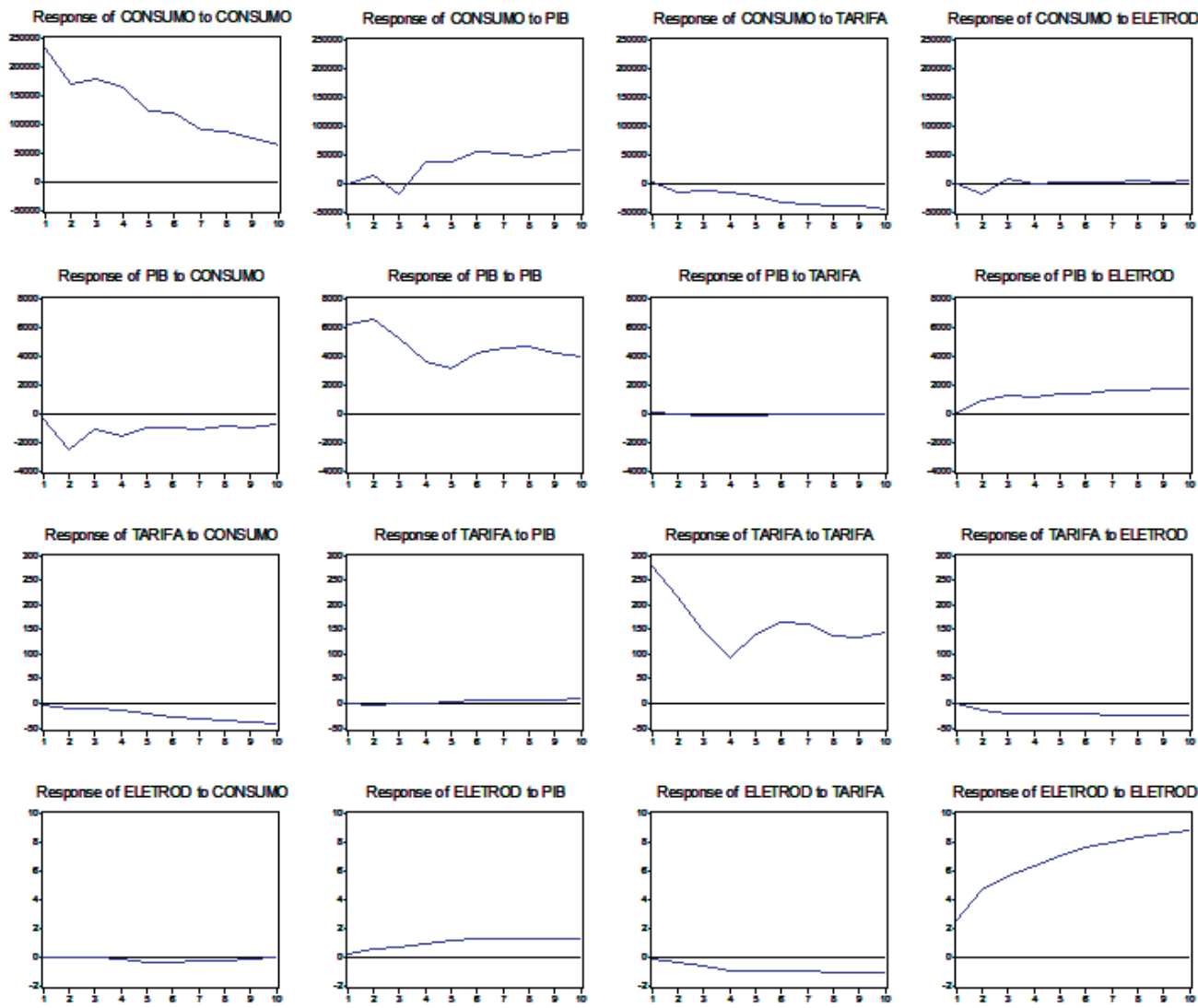

Fonte: Resultados da pesquisa

Outro instrumental disponível pela metodologia VAR é a Análise de Decomposição de Variância (ADV) do erro de previsão que descreve a dinâmica do sistema na abordagem do modelo VAR, o que possibilita auferir que porcentagem da variância de erro de previsão decorre de cada variável endógena ao longo do horizonte de previsão do modelo. É um método que auxilia na identificação da proporção pós-variação total de uma variável, que é resultado de um choque individual nas $\mathrm{k}$ variáveis componentes do modelo. Desse modo, ADV disponibiliza informações sobre a importância relativa de cada inovação sobre as variáveis do sistema (PINTO; VIEIRA, 2008).

Sendo assim, observando as tabelas 8, 9, 10 e 11 é possível verificar a participação de cada variável do modelo na variância da demanda por energia ao longo do 
período de análise. A tabela 8 mostra que, no primeiro ano, o consumo quase total de energia é explicado por ele mesmo, ou seja, independentemente das variáveis PIB, tarifa de energia e preço médio do eletrodoméstico, quase $100 \%$ da demanda não sofre influência dessas variáveis. Após um período de dez anos, percebe-se que as variáveis PIB, tarifa de energia e preço médio do eletrodoméstico influenciam na demanda por energia com participação de $8,1 \%, 8,2 \%$ e $0,3 \%$ respectivamente.

Tabela 8: Decomposição da variância para o consumo

\begin{tabular}{cccccc}
\hline ANOS & ERRO PADRÃO & CONSUMO & PIB & TARIFA & P. ELETROD \\
\hline 1 & 231649.5 & 100.0000 & 0.000000 & 0.000000 & 0.000000 \\
5 & 390552.0 & 96.90501 & 0.795760 & 2.045208 & 0.254022 \\
10 & 470733.0 & 83.33164 & 8.136480 & 8.225935 & 0.305948 \\
\hline
\end{tabular}

Fonte: Resultados da pesquisa

A decomposição da variância para o PIB, na tabela 9, mostra que no primeiro período $98,7 \%$ do PIB é explicado por ele mesmo, e 1,2\% pelo consumo. Já no décimo período $94 \%$ da ADV é explicada pelo PIB, 0,08\% pela tarifa e 3,6\% pelo preço médio do eletrodoméstico.

Tabela 9: Decomposição da variância para o PIB

\begin{tabular}{cccccc}
\hline ANOS & ERRO PADRÃO & CONSUMO & PIB & TARIFA & P. ELETROD \\
\hline 1 & 6482.377 & 1.214249 & 98.78575 & 0.000000 & 0.000000 \\
5 & 14301.80 & 3.421804 & 93.90417 & 0.008102 & 2.665926 \\
10 & 18885.63 & 1.976664 & 94.32142 & 0.080959 & 3.620957 \\
\hline
\end{tabular}

Fonte: Resultados da pesquisa

$\mathrm{Na}$ tabela 10 , a decomposição da variância para a tarifa de energia é explicada por $99,9 \%$ no primeiro período, enquanto que no décimo período a explicação é dada em $95,4 \%$ pela tarifa de energia, $1,1 \%$ do PIB e $0,6 \%$ pelo preço médio do eletrodoméstico.

Tabela 10: Decomposição da variância para tarifa de energia

\begin{tabular}{cccccc}
\hline ANOS & ERRO PADRÃO & CONSUMO & PIB & TARIFA & P. ELETROD \\
\hline 1 & 290.5792 & 0.083974 & $1.71 \mathrm{E}-05$ & 99.91601 & 0.000000 \\
5 & 510.0302 & 0.838314 & 0.218796 & 98.59129 & 0.351600 \\
10 & 680.9925 & 2.723171 & 1.113332 & 95.49832 & 0.665175 \\
\hline
\end{tabular}

Fonte: Resultados da pesquisa

Por fim, na tabela 11 a ADV mostra que no primeiro período 99,6\% é explica- 
da pelo preço médio do eletrodoméstico, 0,08\% do PIB e 0,12\% da tarifa de energia elétrica. Para o décimo período, a ADV é explicada por $98 \%$ pelo preço médio do eletrodoméstico, 0,13\% do PIB e 1,09\% pela tarifa de energia.

Tabela 11: Decomposição da variância para preço médio do eletrod.

\begin{tabular}{cccccc}
\hline ANOS & ERRO PADRÃO & CONSUMO & PIB & TARIFA & P. ELETROD \\
\hline 1 & 2.581411 & 0.182470 & 0.086156 & 0.126587 & 99.60479 \\
5 & 12.81952 & 0.036480 & 0.511580 & 0.793364 & 98.65858 \\
10 & 21.52005 & 0.131365 & 0.747630 & 1.094256 & 98.02675 \\
\hline
\end{tabular}

Fonte: Resultados da pesquisa

Conforme os resultados das tabelas acima, observa-se que a demanda por energia tem uma relativa influência das variáveis dependentes (preço médio do eletrodoméstico, PIB e tarifa de energia), mas estas não são determinantes para explicar o aumento do consumo por energia. Ou seja, independentemente daquelas variáveis, a energia elétrica sofre influência da própria demanda por se tratar de um bem público indispensável para a sociedade e para a atividade econômica de um país.

\section{Conclusões}

O presente trabalho teve como objetivo analisar empiricamente a relação causal entre demanda por energia elétrica e as suas variáveis determinantes: PIB, tarifa de energia e o preço médio dos eletrodomésticos. A hipótese econômica está alicerçada na relação existente entre o consumo de energia elétrica residencial, que possui uma correlação positiva com o PIB e negativa com a tarifa de energia e preços dos eletrodomésticos, que foram investigados neste trabalho.

Desse modo, este trabalho procurou contribuir para as lacunas existentes sobre pesquisas e estudos quantitativos da demanda residencial de energia elétrica a nível nacional, no qual pode evidenciar como está reagindo o consumo de energia de acordo com variáveis ligadas ao desempenho da economia. De modo que esses estudos podem facilitar e/ou direcionar certas políticas públicas para o setor elétrico com objetivo de atender a evolução da demanda por energia da classe residencial, permitindo segurança no abastecimento e bem-estar doméstico para as pessoas.

Foi possível constatar que o Brasil tem na hidrelétrica a sua principal fonte de produção de energia e está passando por problemas operacionais na oferta de energia devido ao subinvestimento no setor, ocasionado desde a época do nacional desenvolvimentismo. No entanto, muitos são os desafios, pois a falta de investimento ao longo dos anos e a característica sazonal no regime de chuvas da fonte hídrica associada às restrições das leis ambientais, exige cada vez mais fontes alternativas de energia para atender à demanda.

Os resultados econométricos mostraram, por meio do vetor de cointegração, 
que as variações no preço dos eletrodomésticos têm pouca significância na determinação do consumo de energia elétrica residencial. Para longo prazo, as elasticidades preço e renda da demanda por energia elétrica apresentam sinais esperados. Sendo que, uma variação positiva no PIB impacta mais a demanda por energia do que uma redução do preço da tarifa.

Nas funções de impulso resposta, pelo teste de causalidade de Granger, demonstram que as variáveis independentes influenciam no comportamento da demanda por eletricidade na classe residencial. Porém, vale lembrar que energia elétrica é um bem de consumo bastante importante na vida das pessoas, o que leva a considerar que essas pessoas são dependentes do fornecimento de energia para o bem-estar doméstico e conforto social.

Como o consumo de energia apresenta tendência de crescimento ao longo dos anos, a oferta de energia precisa sempre estar a frente da demanda. Dessa maneira, a introdução de políticas públicas de incentivo a produção de outras fontes energéticas tornam-se essenciais para uma maior diversificação da matriz elétrica. Numa perspectiva de longo prazo, o Brasil pode planejar e investir também em política industrial com participação de instituições públicas e privadas para desenvolvimento de $\mathrm{P} \& \mathrm{D}$ com objetivo de criar conhecimento e tecnologia nacional para fontes alternativas, como é o caso da energia eólica e outras fontes com o objetivo de expandir a oferta de energia.

Por fim, este trabalho não pretende esgotar a temática abordada, uma vez que o setor elétrico tem diversas áreas de pesquisa, além de ser fundamental para o crescimento e desenvolvimento econômico. Assim, espera-se que futuras pesquisas possam investigar não só a demanda nacional por energia na classe residencial, mas também as demandas das classes comercial e industrial.

\section{Referências}

ABREU, Y. V. A reestruturação do setor elétrico brasileiro: Questões e Perspectivas. Dissertação de Mestrado apresentada ao Programa Interunidades de Pós-graduação em Energia da Universidade de São Paulo, São Paulo, 1999.

AGÊNCIA NACIONAL DE ENERGIA ELÉTRICA (ANEEL). Atlas de Energia Elétrica do Brasil, $3^{a}$ edição. Brasília, 2008. Disponível em: http://www.aneel.gov. $\mathrm{br} /$ arquivos/pdf/atlas_capa_sumario.pdf >. Acesso em: 3 mar de 2016.

. Banco de Informações de Geração: capacidade de geração do Brasil. Disponível em: http://www2.aneel.gov.br/aplicacoes/capacidadebrasil/capacidadebrasil. cfm. Acesso em: 30 Jun 2017.

ANDRADE, T. \& LOBÃO, W. (1997). Elasticidade-renda e preço da demanda residencial de energia elétrica no Brasil. Texto para discussão n. 489, RJ, IPEA. 
BARDELIN, C. E. A. Os efeitos do Racionamento de Energia Elétrica ocorrido no Brasil em 2001 e 2002 com ênfase no Consumo de Energia Elétrica. 2004. 112 P. Dissertação (Mestrado em Engenharia). Escola Politécnica da USP, São Paulo, SP. crossref https://doi.org/10.11606/D.3.2004.tde-23062005-084739

CASTRO, N. J. Avanços na reestruturação do setor de energia elétrica. IFE: Informe Eletrônico, Rio de Janeiro, n. 1081, 2 p. 31 mar. 2003. Disponível em: < http://www. provedor.nuca.ie.ufrj.br/eletrobras/artigos/castro6.htm>. Acesso em: 18 jul 2016.

ELETROBRAS. Departamento de Estudos Energéticos e Mercados (DEM). Plano Decenal de Expansão 2003/2012. Janeiro de 2003. Disponível em: http://www.eletrobras.com/elb/data/Pages/LUMIS293E16C4PTBRIE.htm. Acesso em: 10 jul de 2016.

EMPRESA DE PESQUISA ENERGÉTICA - EPE. Boletim de Conjuntura energética: $2^{\circ}$ trimestre 2011. Rio de Janeiro. Disponível em: http://www.epe.gov.br/ mercado/Documents/S\%C3\%A9rie\%20Estudos\%20de\%20Energia/20111226_1. pdf. Acesso em 12 abr. 2016.

ENGLE, R. F.; GRANGER, C.W. Co-integration and error-correction: representation, estimation and testing. Econometrica, v. 55, p. 251-76, 1987. Disponível em: <http://ideas.repec.org/a/ecm/emetrp/v55y1987i2p251-76.html>. Acesso em: 12 abr de 2016.

GUJARATI, D. Econometria Básica. Rio de Janeiro: Elsevier, 2.ed. 2006.

INSTITUTO DE PESQUISA ECONÔMICA APLICADA - IPEA. Disponível em: < http://www.ipeadata.gov.br/Default.aspx>. Acesso em: 30 de jun 2017

IRFFI, G. et al. Previsão da Demanda por Energia Elétrica para Classes de Consumo na Região Nordeste, usando OLS Dinâmico e Mudança de Regime. Revista de Economia Aplicada - USP, São Paulo, v. 13, n. 1, p. 69-98, Jan./Mar. 2009.

JOHANSEN, S. Statistical analysis of cointegration vectors. Journal of Economics Dynamics and Control, v. 12, p.231-254, 1988. crossref https://doi. org/10.1016/0165-1889(88)90041-3

MATTOS, L.B, LIMA, J.E, Demanda residencial de energia elétrica em Minas Gerais: 1970-2002. Nova Economia: FACE/UFMG. Disponível em: http://www.scielo.br/pdf/neco/v15n3/v15n3a02.pdf. Acesso em: 08 jun. 2016.

MODIANO, E. M. Elasticidade renda e preços da demanda de energia elétrica no Brasil. Rio de Janeiro: Departamento de Economia da PUC, 1984. (Texto para Discussão, n. 68). 
MORETTIN, P. A., TOLOI, C. M. C. Análise de Séries Temporais. 2. ed. São Paulo: Edgard Blücher, 2006.

OLIVEIRA NETO. C. R. ENERGIA EÓLICA E DESENVOLVIMENTO NO TERCEIRO MILENNIO: reflexões a partir do Brasil, Nordeste e Rio Grande do Norte. 2016. Dissertação de Mestrado do Programa de Pós Gradução em Economia da Universidade Federal do Rio Grande do Norte. Disponível em: https://repositorio.ufrn.br/jspui/browse type $=$ author\&value $=$ Oliveira + Neto $\% 2 \mathrm{C}+$ Calisto + Rocha+de. Acesso em: 30 de Jun 2017.

PINTO, A. C. B.; VIEIRA, F. V. Câmbio flexível e metas de inflação em países selecionados da América Latina: análise de vetores auto-regressivos (VAR). Revista Ensaios FEE. v. 29. n.1 Porto Alegre. 2008.

SAED, A. A. J. Estimation of total energy consumption in Jordan 1980-1999: Evidence from co-integration and stock. Journal of Economic E Administrative Sciences, v. 20, n. 1, p. 71-82, 2004. crossref https://doi.org/10.1108/10264116200400004

SILVA, N. F. Fontes de Energia Renováveis Complementares na Expansão do Setor Elétrico Brasileiro: O Caso da Energia Eólica. 2006. Tese (Doutorado) em Ciências em Planejamento Energético. Universidade Federal do Rio de Janeiro - COPPE, Rio de Janeiro. 2006. Disponível em: http://www.ppe.ufrj.br/ppe/production/tesis/nfsilva.pdf. Acesso em: 21 jun 2016.

VARIAN, H.R. Microeconomia: princípios básicos. $7^{\circ}$ ed. Rio de Janeiro: Campus, 2006.

U.S. EIA - U.S. ENERGY INFORMATION ADMINISTRATION. China International energy data and analysis, 2015. Disponível em: https://www.eia.gov/beta/ international/analysis_includes/countries_long/China/china.pdf. Acesso em: 29 Jun. 2017.

. Electricity Explained, 2014. Disponível em: http://www.eia.gov/energyex-

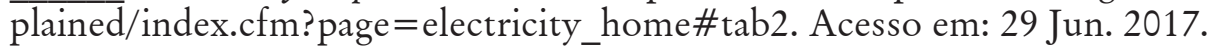

Recebido em 29.09.16

Aprovado em 06.09.17 
